\title{
Correlation of Fecal Markers with Magnifying Endoscopic Stratification in Patients with Ulcerative Colitis Who Are in Clinical Remission
}

\author{
Shoichiro Mine ${ }^{1}$, \\ Fuminao Takeshima ${ }^{1}$, \\ Yuko Akazawa ${ }^{1}$, \\ Kayoko Matsushima ${ }^{1}$ \\ Hitomi Minami ${ }^{1}$, \\ Naoyuki Yamaguchi ${ }^{1}$, \\ Ken Ohnita ${ }^{1}$, \\ Hajime Isomoto ${ }^{2}$ \\ Kazuhiko Nakao ${ }^{1}$
}

1. Department of Gastroenterology and Hepatology, Graduate School of Biomedical Science, Nagasaki University, Nagasaki, Japan

2. Division of Medicine and Clinical Science, Tottori University Faculty of Medicine,

Tottori, Japan

Authors' contributions:

SM participated in the data collection, data analysis, and manuscript drafting. FT participated in the study design, data collection, data analysis, and manuscript drafting. YA participated in the data analysis and manuscript drafting. KM and HM participated in the data collection and data analysis. NY, KO, and HI participated in the data collection. KN gave final approval of the manuscript to be published. All authors read and approved the final manuscript.

Address for correspondence author: Shoichiro Mine, MD Nagasaki University Hospital 1-7-1, Sakamoto, Nagasaki City, Nagasaki Japan, Zip 852-8501

E-mail: hyotan999@yahoo.co.jp

Tel:+81-095-819-7481

Fax:+81-095-819-7482 


\section{Key Words}

Fecal marker - Calprotectin - Lactoferrin - Ulcerative Colitis Endoscopic Index of Severity $\cdot$ Magnifying endoscopic stratification $\cdot$ Mayo endoscopic subscore $\cdot$ Ulcerative colitis in clinical remission $\cdot$ Right colon inflammation 


\section{Abstract}

Background: Maintenance of mucosal healing is recommended during the treatment of ulcerative colitis (UC). However, symptoms of UC often do not reflect mucosal disease activity. Fecal markers such as calprotectin, lactoferrin, and hemoglobin have been reported to correlate well with the Mayo endoscopic subscore (MES) and are being considered alternative monitoring tools in endoscopy. Ulcerative Colitis Endoscopic Index of Severity (UCEIS) is a new and more detailed endoscopic scoring system compared to MES. Furthermore, magnifying endoscopic stratification (ME) based on alterations in the mucosal surface pit patterns is noted in UC. However, the association between fecal markers and UCIES and magnifying endoscopy is relatively unexplored.

Summary: This study investigated the association between the aforementioned fecal markers and MES, UCEIS, and ME in patients with UC in clinical remission. This prospective study included 60 patients with UC in clinical remission who underwent colonoscopy at the Nagasaki University Hospital between June 2015 and November 2016. A significant correlation was observed between MES and all fecal markers. Notably, the fecal markers correlated well with UCEIS (calprotectin Spearman's correlation coefficient $[\mathrm{r}]=0.54, \mathrm{P}<0.0001$; lactoferrin $\mathrm{r}=0.56, \mathrm{P}<0.0001$; and hemoglobin 
$\mathrm{r}=0.43, \mathrm{P}<0.001$ ). Furthermore, $\mathrm{ME}$ findings correlated significantly with calprotectin $(\mathrm{r}=0.50, \mathrm{P}=0.0002)$ and lactoferrin $(\mathrm{r}=0.46, \mathrm{P}=0.0006)$ levels and slightly with hemoglobin $(\mathrm{r}=0.28, \mathrm{P}=0.043)$ levels. Moreover, each cut-off level of fecal calprotectin, lactoferrin, or hemoglobin had a high sensitivity and specificity for the detection of MES=0, UCEIS $=0, \mathrm{ME}=\mathrm{A}$, for predicting mucosa healing.

Key Messages: Fecal markers correlated not only with MES but also with UCEIS and ME and should be useful for monitoring patients with UC in clinical remission. 


\section{Introduction}

Ulcerative colitis (UC) is a chronic, idiopathic, inflammatory bowel disease that causes diffuse inflammatory mucosal damage, causing erosions and ulcers in the colon, especially in the rectum. The disease has a lifelong clinical course characterized by the repeat of relapse and remission. The main purpose of therapy in UC is to suppress mucosal inflammation and induce and sustain clinical remission. Recent evidence recommends sustained endoscopic remission, which is associated with improved outcomes for UC [1, 2]. Importantly, patient symptoms often do not reflect mucosal disease activity. Consequently, to evaluate disease activity and therapeutic efficacy, clinical symptoms and laboratory and endoscopic findings need to be monitored routinely in clinical practice [3]. Currently, colonoscopy is the most effective means of estimating of UC severity. However, colonoscopy is burdensome, invasive, and costly for patients, and requires the diagnostic skills of the endoscopist. Because frequent colonoscopy is not realistic for UC patients, the development of non-invasive surrogate markers is necessary.

Fecal markers such as calprotectin, lactoferrin, and hemoglobin have been reported to correlate well with the Mayo endoscopic subscore (MES) and are being considered alternative monitoring tools in endoscopy [4-8]. Calprotectin is a calcium-binding 
protein and accounts for up to $60 \%$ of cytoplasmic proteins in neutrophils [6], and is mainly derived from neutrophils. Fecal calprotectin concentration is reported to be associated with the concentration of neutrophils in the colonic mucosa [9]. It is convenient to handle since calprotectin is stable in the feces for up to 1 week at room temperature $[6,10]$. Lactoferrin is an iron-binding glycoprotein secreted from most mucosal membranes and is also a major component of neutrophils $[5,11,12]$. When inflammation occurs in the intestinal tract, leucocytes enter the mucosa and this may increase the excretion of lactoferrin in stool. Lactoferrin is also convenient to handle since it is relatively stable in the stool for up to 4 days $[13,14]$.

The Ulcerative Colitis Endoscopic Index of Severity (UCEIS) is a newly proposed and more detailed endoscopic scoring system compared to MES [15]. In addition to the more detailed scoring of the extent of ulcers compared to MES, UCIES includes the degree of vascular appearance. Indeed, it has been reported that UCEIS precisely reflects clinical outcomes and predicts the medium-to-long-term prognosis in UC patients on induction therapies [16]. However, only few studies have examined the association between fecal markers and UCEIS [17]. Furthermore, we recently proposed a magnifying endoscopic stratification (ME) based on alterations in the mucosal surface pit patterns, which are associated not only with the Matts endoscopic score but also 
with pathognomonic microscopic features of mucosal inflammation [18]. Notably, we also demonstrated its utility in predicting relapse in patients with UC [18]. However, the association between fecal surrogate markers and UCIES or ME has not been studied.

The aim of our study was to investigate the correlation of fecal calprotectin, lactoferrin, and hemoglobin levels with MES, UCEIS and ME in patients with UC in clinical remission.

\section{Material and Methods}

\section{Study Design and Patients}

This prospective study included 60patients with UC in clinical remission who underwent colonoscopy at the Nagasaki University Hospital between June 2015 and November 2016. The inclusion criteria were as follows: UC patients diagnosed with established criteria, aged $\geq 20$ years, and in clinical remission. The exclusion criteria were active UC, pregnancy, liver, kidney, and heart disease, and other severe complications. Clinical remission was defined as a partial UC disease activity index of $\leq 2$ and a bloody stool score of 0 [19].

Measurement of Fecal Calprotectin, Lactoferrin, and Hemoglobin Levels 
Stool samples were collected before colonoscopy on the same day. The collected feces were stored at room temperature and immediately analyzed at the Kyoto Medical Science Laboratory (Kyoto, Japan). Fecal lactoferrin was measured using sandwich enzyme-linked immunosorbent assay, previously described in detail [20]. Fecal calprotectin level was measured using an enzyme-linked immunosorbent assay kit (Immunodiagnostik AG, Benshem, Germany). Hemoglobin level was measured using the Nescauto hemo plus (Alfresa Pharma Corporation, Japan).

Ulcerative Colitis Endoscopic Index of Severity

MES, UCEIS, and ME were evaluated during the same session of colonoscopy. Scores and classifications were determined by the agreement of two experienced endoscopists. The UCEIS score is based on a visual analog scale represented by incorporating a combination of 3 elements: vascular pattern (2 levels), bleeding (3 levels), and erosions and ulcers (3 levels). Via colonoscopy, the worst state of the colon is identified, and the score may be calculated by adding the scores from each section ranging from 0 (normal) to 8 (worst) [17].

Magnifying Endoscopic Stratification

ME observations were performed at the location where the most severe inflammation was detected during white light examination. For cases without apparent inflammation, 
ME was performed in the rectal mucosa. The endoscopy system consisted of a light source (CLV-260SL; Olympus Optical Co., Tokyo, Japan), a colonoscope (PCF-Q260AZI; Olympus), and a processor (CV-260SL; Olympus). On insertion of the colonoscope, luminal cleansing was performed using water with dimethicone and pronase. After standard observation, in the rectal mucosa, ME was performed following vital staining including $0.05 \%$ crystal violet. ME findings were classified into 4 grades: ME-A, regular arrangement of normal round-to-oval pits; ME-B, irregular arrangement of the round-to-oval pits with/without enlarged spaces between the even crypts; ME-C, irregular pits in size and shape with a more irregular arrangement of the pits compared to ME-B; and ME-D, destruction and disappearance of pits, as previously described by Isomoto et al [18].

\section{Statistical Analysis}

Statistical analyses were performed using JMP version 11.0 for Windows (SAS Institute, Cary, NC, USA). Sensitivity and specificity with $95 \%$ confidence intervals for finding MES, UCEIS, and ME levels were established according to calprotectin, lactoferrin, and hemoglobin results. We analyzed the receiver operating characteristic (ROC) curve and the area under the curve (AUC) to evaluate the appropriate cut-off values for calprotectin, lactoferrin, and hemoglobin. Moreover, we conducted the Spearman's rank 
correlation test to clarify the correlation between calprotectin, lactoferrin, and hemoglobin levels and MES, UCEIS, and ME. p-values < 0.05 were considered statistically significant.

Ethical Considerations

The study was approved by the Nagasaki University Ethics Committee (Number: 16020830) and was conducted in accordance with the Helsinki Declaration. Informed consent was acquired from all patients in advance regarding the use of their fecal samples and clinical data for this study.

\section{Results}

Clinical Characteristics of Patients (Table 1)

The total number of 60 patients consisted of 35 men and 25 women. The median patient age was 51. Among the 60 UC patients, 40 (67\%) had extensive colitis, $13(22 \%)$ had left-sided colitis, and $7(12 \%)$ had proctitis. For remission maintenance therapy, salazosulfapyridine was used in 5 patients, mesalamine in 43 patients, azathioprine/mercaptopurine in 13 patients, infliximab/adalimumab in 5 patients, and 6 patients received no medication.

\section{Colonoscopic Findings}


Of the 60 cases, 18 cases (30\%) had a MES score of 0,27 cases (45\%) had a MES score of 1 , and $15(25 \%)$ cases had a MES score of 2 . Seventy percent (42/60) of patients in clinical remission had ongoing endoscopic inflammation (MES $\geq 1$ ).

Of the 60 cases, 16 cases $(27 \%)$ had a UCEIS score of 0,20 cases $(33 \%)$ had a UCEIS score of $1,7(11 \%)$ cases had a UCEIS score of $2,8(13 \%)$ cases had a UCEIS score of 3,7 (12\%) cases had a UCEIS score of 4, $1(1.7 \%)$ case had a UCEIS score of 5, and $1(1.7 \%)$ case had a UCEIS score of 6.

Of the 60 cases, 52 cases underwent ME. According to ME stratification, there were 21 cases of ME-A, 17 cases of ME-B, 12 cases of ME-C, and 2 cases of ME-D (Table 2).

Relationship between Calprotectin, Lactoferrin, Hemoglobin, and Colonoscopic Findings

A significant correlation was observed between MES and all fecal markers (Spearman's correlation coefficient $[\mathrm{r}]$ for calprotectin $=0.34, \mathrm{P}<0.01$; lactoferrin $\mathrm{r}=0.45, \mathrm{P}<0.01$; and hemoglobin $\mathrm{r}=0.38, \mathrm{P}<0.01$ ) and between UCEIS and all fecal markers (calprotectin $\mathrm{r}=0.54, \mathrm{P}<0.0001$; lactoferrin $\mathrm{r}=0.56, \mathrm{P}<0.0001$; and hemoglobin $\mathrm{r}=$ 0.43, $\mathrm{P}<0.001$ ) (Figure 1a). Furthermore, ME findings correlated significantly with calprotectin and lactoferrin levels and slightly with hemoglobin levels (calprotectin $\mathrm{r}=$ $0.50, \mathrm{P}<0.001$; lactoferrin $\mathrm{r}=0.46, \mathrm{P}<0.001$; and hemoglobin $\mathrm{r}=0.28, \mathrm{P}=0.043$ ) 
(Figure 1b). In addition, a significant correlation was observed between ME and UCEIS $(\mathrm{r}=0.74, \mathrm{P}<0.0001)$. These results suggest that fecal markers correlate not only with MES but also with UCEIS and ME.

Receiver Operating Curve Analysis and the Predictive Ability of Fecal Markers

Calprotectin, at a cut-off of $201 \mu \mathrm{g} / \mathrm{ml}$, had a sensitivity of $71 \%$ and specificity of $78 \%$ for the detection of MES=0 (prediction of mucosal healing) (AUC 0.77). Lactoferrin (cut-off $78.3 \mathrm{ng} / \mathrm{ml}$ ) had a sensitivity of $60 \%$ and specificity of $89 \%$ for the detection of MES=0 (AUC 0.73). Hemoglobin (cut-off $34 \mathrm{ng} / \mathrm{ml}$ ) had a sensitivity of $64 \%$ and specificity of 89\% for the detection of MES=0 (AUC 0.76). Calprotectin, at a cut-off of $201 \mu \mathrm{g} / \mathrm{ml}$, had a sensitivity of $70 \%$ and specificity of $81 \%$ for the detection of UCEIS=0 (prediction of mucosal healing) (AUC 0.76). Lactoferrin (cut-off $78.3 \mathrm{ng} / \mathrm{ml}$ ) had a sensitivity of $57 \%$ and specificity of $88 \%$ for the detection of UCEIS=0 (AUC0.71). Hemoglobin (cut-off 31 $\mathrm{ng} / \mathrm{ml}$ ) had a sensitivity of $64 \%$ and specificity of $88 \%$ for the detection of UCEIS $=0$ (AUC 0.74) (Figure 2a). Calprotectin, at a cut-off of $280 \mu \mathrm{g} / \mathrm{ml}$, had a sensitivity of $68 \%$ and specificity of $86 \%$ for the detection of $\mathrm{ME}=\mathrm{A}$ (prediction of mucosal healing) (AUC 0.78). Lactoferrin (cut-off $43.6 \mathrm{ng} / \mathrm{ml}$ ) had a sensitivity of $71 \%$ and specificity of $76 \%$ for the detection of $\mathrm{ME}=\mathrm{A}$ (AUC 0.77). Hemoglobin (cut-off $34 \mathrm{ng} / \mathrm{ml}$ ) had a sensitivity of $71 \%$ and specificity of $86 \%$ for the detection of $\mathrm{ME}=\mathrm{A}$ (AUC 0.78) (Figure 2b). These 
results suggest that fecal markers are useful for monitoring patients with UC in clinical remission.

\section{Detection of the Inflammation in Right-Side Colon}

In 10 of the 40 patients with extensive colitis, endoscopic inflammation remained only in the right side of the colon. In these 10 cases, calprotectin, lactoferrin, and hemoglobin, at a cut-off value (for detecting UCEIS=0 i.e. the prediction of mucosal healing) of 201 $\mu \mathrm{g} / \mathrm{ml}, 78.3 \mathrm{ng} / \mathrm{ml}$, and $31 \mathrm{ng} / \mathrm{ml}$, respectively, had a sensitivity of 100\% (10/10), 70\% (7/10), and 50\% (5/10), respectively. No significant difference was found between the markers. This result suggests that calprotectin could be a highly sensitive tool for detecting inflammation in the right side of the colon.

\section{Discussion}

In this study, although all the patients were in clinical remission, 70\% (42/60) of patients had ongoing endoscopic inflammation, including 25\% cases with a MES score of 2 . Our results suggest that patients with active intestinal inflammation occasionally have no symptoms or may fail to report symptoms. Therefore, monitoring symptoms alone lead to under-treatment of the disease and a higher likelihood of clinical recurrence. Recently, the importance of achieving mucosal healing, rather than 
symptoms elimination alone, has been recognized [21]. Moreover, it has been reported that patients who achieve mucosal healing have lower rates of hospitalization and a lower risk of colectomy [1, 2]. Furthermore, in UC patients, a prolonged disease course and immunosuppressive therapy increase the risk for emerging malignancies compared to those who have a normal colon [22-24].

Recently, fecal markers have received attention as means for the noninvasive monitoring of disease activity. Several studies have reported that fecal markers such as calprotectin, lactoferrin, and hemoglobin correlate well with MES [4, 25, 26], but only few studies have examined the association between fecal markers and UCEIS [17]. In this study, we confirmed the correlation of fecal calprotectin, lactoferrin, and hemoglobin with MES or UCEIS in patients with UC in clinical remission. Surface pit pattern classification with $\mathrm{ME}$ is known to have a good association with the histopathological diagnosis of colorectal tumor [18]. Similarly, recent studies have shown that ME findings are significantly correlated with histological grades [27, 28]. Of note, it is reported that $\mathrm{ME}$ could be useful in assessing microscopic inflammatory features of UC that cannot be determined by white light colonoscopic examination [27, 28]. The use of ME in UC is relatively new and different ME classifications have been proposed to date $[18,27,28]$. Further studies are required to determine the 
classifications that most accurately represent and predict the patient's clinical status and outcome. This study is the first to report that fecal calprotectin and lactoferrin levels are significantly correlated with $\mathrm{ME}$ grade in patients with UC in clinical remission. Moreover, the cut-off levels of fecal calprotectin, lactoferrin, or hemoglobin had a high sensitivity and specificity for the detection of MES $=0$, UCEIS $=0, \mathrm{ME}=\mathrm{A}$.

Therefore, fecal markers should be considered as alternative monitoring tools in endoscopy and could provide an opportunity for recommending colonoscopy to patients with UC in clinical remission.

In the 10 cases with extensive colitis involving macroscopic inflammation on the right side of the colon alone, fecal calprotectin, lactoferrin, and hemoglobin, at a cut-off value for detecting UCEIS=0, had a sensitivity of 100\% (10/10), 70\% (7/10), and $50 \%(5 / 10)$, respectively. It is reported that hemoglobin tends to lose antigenicity because of its degradation by fecal bacteria and digestive enzymes [29]. Moreover, the stability of calprotectin in the feces is also reported to be greater than that of lactoferrin $[6,14]$. Our results suggest that calprotectin could have a superior sensitivity, compared to other fecal markers, for the detection of inflammation on the right side of the colon, although the small sample size may have masked the true differences.

Reports show that in patients with UC, regulation of the 5-aminosalicylic acid 
(ASA) dose, with the monitoring of fecal calprotectin levels, resulted in significantly lower relapse rates compared to the control group [30, 31]. Isomoto et al. demonstrated significant differences in the percentages of relapse of UC among the ME grades retrospectively. Our prospective study is ongoing, to analyze whether ME findings can predict relapse in UC. When future relapse of UC is predicted by fecal markers and colonoscopy findings, it may be possible to take preventive measures such as increasing 5-ASA dosage before symptoms appear and, thus, exclude complications and the progression to intractable cases.

In conclusion, our analysis demonstrated that fecal markers correlated not only with MES but also with UCEIS and ME and may be useful for monitoring patients with UC in clinical remission. Moreover, we suggest that calprotectin is a highly sensitive tool for detecting macroscopic inflammation on the right side of the colon. Additional prospective studies are required to ascertain whether fecal markers can be used as an alternative to endoscopy with biopsy for improving treatment optimization and outcomes.

\section{Acknowledgements}

We would like to express our gratitude to Dr. K. Hashiguchi, Dr. M. Kitayama, Dr. K. 
Ogihara, Dr. T. Akashi, Dr. M. Tabuchi, Dr. J. Shiota, Dr. R. Iyama, Dr. A. Kuwahara, Dr.

W. Tanaka, and Dr. T. Hori for providing the clinical data. We would also like to thank the Kyoto Medical Science Laboratory (Kyoto, Japan) for measuring the fecal calprotectin, lactoferrin, and hemoglobin, and Editage (Tokyo, Japan) for the English language review.

\section{Conflict of Interests}

The authors declare that they have no conflict of interests regarding the publication of this manuscript.

\section{References}

1. Ardizzone S, Cassinotti A, Duca P, et al. Mucosal healing predicts late outcomes after the first course of corticosteroids for newly diagnosed ulcerative colitis. Clin Gastroenterol Hepatol 2011;9(6):483-89.e3. doi: 10.1016/j.cgh.2010.12.028 [published Online First: 2010/12/31]

2. Ferrante M, Vermeire S, Fidder H, et al. Long-term outcome after infliximab for refractory ulcerative colitis. $J$ Crohns Colitis 2008;2(3):219-25. doi: 10.1016/j.crohns.2008.03.004 [published Online First: 2008/05/16]

3. Nikolaus S, Schreiber S. Diagnostics of inflammatory bowel disease. Gastroenterology 2007;133(5):1670-89. doi: 10.1053/j.gastro.2007.09.001

4. Takashima S, Kato J, Hiraoka S, et al. Evaluation of Mucosal Healing in Ulcerative Colitis by Fecal Calprotectin Vs. Fecal Immunochemical Test. Am J Gastroenterol 
2015;110(6):873-80. doi: 10.1038/ajg.2015.66

5. Walker TR, Land ML, Kartashov A, et al. Fecal lactoferrin is a sensitive and specific marker of disease activity in children and young adults with inflammatory bowel disease. $J$ Pediatr Gastroenterol Nutr 2007;44(4):414-22. doi: 10.1097/MPG.0b013e3180308d8e

6. Bunn SK, Bisset WM, Main MJ, et al. Fecal calprotectin: validation as a noninvasive measure of bowel inflammation in childhood inflammatory bowel disease. $J$ Pediatr Gastroenterol Nutr 2001;33(1):14-22.

7. Kanmura S, Hamamoto H, Morinaga Y, et al. Fecal Human Neutrophil Peptide Levels Correlate with Intestinal Inflammation in Ulcerative Colitis. Digestion 2016;93(4):300-8. doi: 10.1159/000446210 [published Online First: 2016/05/26]

8. D'Angelo F, Felley C, Frossard JL. Calprotectin in Daily Practice: Where Do We Stand in 2017? Digestion 2017;95(4):293-301. doi: 10.1159/000476062 [published Online First: 2017/05/17]

9. Voganatsi A, Panyutich A, Miyasaki KT, et al. Mechanism of extracellular release of human neutrophil calprotectin complex. J Leukoc Biol 2001;70(1):130-4.

10. Johne B, Fagerhol MK, Lyberg T, et al. Functional and clinical aspects of the myelomonocyte protein calprotectin. Mol Pathol 1997;50(3):113-23.

11. Baveye S, Elass E, Mazurier J, et al. Lactoferrin: a multifunctional glycoprotein involved in the modulation of the inflammatory process. Clin Chem Lab Med 1999;37(3):281-6. doi: 10.1515/CCLM.1999.049

12. Baynes RD, Bezwoda WR. Lactoferrin and the inflammatory response. Adv Exp Med Biol 1994;357:133-41.

13. Kane SV, Sandborn WJ, Rufo PA, et al. Fecal lactoferrin is a sensitive and specific marker in identifying intestinal inflammation. Am $J$ Gastroenterol 2003;98(6):1309-14. doi: 10.1111/j.1572-0241.2003.07458.x

14. Sugi K, Saitoh O, Hirata I, et al. Fecal lactoferrin as a marker for disease activity in inflammatory bowel disease: comparison with other neutrophil-derived proteins. $\mathrm{Am}$ J Gastroenterol 1996;91(5):927-34.

15. Travis SP, Schnell D, Krzeski P, et al. Reliability and initial validation of the ulcerative colitis endoscopic index of severity. Gastroenterology 2013;145(5):987-95. doi: 10.1053/j.gastro.2013.07.024 [published Online First: 2013/07/25]

16. Ikeya K, Hanai H, Sugimoto K, et al. The Ulcerative Colitis Endoscopic Index of Severity More Accurately Reflects Clinical Outcomes and Long-term Prognosis than the Mayo Endoscopic Score. J Crohns Colitis 2016;10(3):286-95. doi: 10.1093/ecco-jcc/jjv210 [published Online First: 2015/11/17] 
17. Taghvaei T, Maleki I, Nagshvar F, et al. Fecal calprotectin and ulcerative colitis endoscopic activity index as indicators of mucosal healing in ulcerative colitis. Intern Emerg Med 2015;10(3):321-8. doi: 10.1007/s11739-014-1144-x [published Online First: 2014/11/04]

18. Isomoto H, Uehara R, Hayashi T, et al. Magnifying Endoscopic Findings Can Predict Clinical Outcome during Long-Term Follow-Up of More Than 12 Months in Patients with Ulcerative Colitis. Gastroenterol Res Pract 2013;2013:671576. doi: 10.1155/2013/671576 [published Online First: 2013/10/02]

19. Yamamoto T, Shiraki M, Bamba T, et al. Fecal calprotectin and lactoferrin as predictors of relapse in patients with quiescent ulcerative colitis during maintenance therapy. Int $J$ Colorectal Dis 2014;29(4):485-91. doi: 10.1007/s00384-013-1817-3 [published Online First: 2013/12/17]

20. Sunwoo H, Gujral N, Suresh M. Competitive and double antibody sandwich ELISA for the quantification of lactoferrins by using monoclonal and chicken egg yolk IgY antibodies. $\boldsymbol{J}$ Immunoassay Immunochem 2011;32(2):79-92. doi: $10.1080 / 15321819.2010 .543218$

21. Rosenberg L, Lawlor GO, Zenlea T, et al. Predictors of endoscopic inflammation in patients with ulcerative colitis in clinical remission. Inflamm Bowel Dis 2013;19(4):779-84. doi: 10.1097/MIB.0b013e3182802b0e

22. Rutter M, Saunders B, Wilkinson K, et al. Severity of inflammation is a risk factor for colorectal neoplasia in ulcerative colitis. Gastroenterology 2004;126(2):451-9.

23. Rutter MD, Saunders BP, Wilkinson KH, et al. Cancer surveillance in longstanding ulcerative colitis: endoscopic appearances help predict cancer risk. Gut 2004;53(12):1813-6. doi: 10.1136/gut.2003.038505

24. Madanchi M, Zeitz J, Barthel C, et al. Malignancies in Patients with Inflammatory Bowel Disease: A Single-Centre Experience. Digestion 2016;94(1):1-8. doi: 10.1159/000447259 [published Online First: 2016/06/18]

25. Turner D, Leach ST, Mack D, et al. Faecal calprotectin, lactoferrin, M2-pyruvate kinase and S100A12 in severe ulcerative colitis: a prospective multicentre comparison of predicting outcomes and monitoring response. Gut 2010;59(9):1207-12. doi: 10.1136/gut.2010.211755

26. Theede K, Holck S, Ibsen P, et al. Level of Fecal Calprotectin Correlates With Endoscopic and Histologic Inflammation and Identifies Patients With Mucosal Healing in Ulcerative Colitis. Clin Gastroenterol Hepatol 2015;13(11):1929-36.e1. doi: 10.1016/j.cgh.2015.05.038 [published Online First: 2015/06/04]

27. Fujiya M, Saitoh Y, Nomura M, et al. Minute findings by magnifying colonoscopy are 
useful for the evaluation of ulcerative colitis. Gastrointest Endosc 2002;56(4):535-42. doi: 10.1067/mge.2002.127101

28. Kunihiro M, Tanaka S, Sumii M, et al. Magnifying colonoscopic features of ulcerative colitis reflect histologic inflammation. Inflamm Bowel Dis 2004;10(6):737-44.

29. Saitoh O, Kojima K, Kayazawa M, et al. Comparison of tests for fecal lactoferrin and fecal occult blood for colorectal diseases: a prospective pilot study. Intern Med 2000;39(10):778-82.

30. Lasson A, Öhman L, Stotzer PO, et al. Pharmacological intervention based on fecal calprotectin levels in patients with ulcerative colitis at high risk of a relapse: A prospective, randomized, controlled study. United European Gastroenterol $J$ 2015;3(1):72-9. doi: 10.1177/2050640614560785

31. Mao R, Xiao YL, Gao X, et al. Fecal calprotectin in predicting relapse of inflammatory bowel diseases: a meta-analysis of prospective studies. Inflamm Bowel Dis 2012;18(10):1894-9. doi: 10.1002/ibd.22861 [published Online First: 2012/01/11] 


\section{Figure legends}

Figure 1a. Correlation between UCEIS and fecal markers

The relationships between calprotectin, lactoferrin, hemoglobin, and UCEIS were analyzed. A significant correlation was observed between UCEIS and all fecal markers (Spearman's rank correlation coefficient for calprotectin $\mathrm{r}=0.54, \mathrm{P}<0.0001$; lactoferrin $\mathrm{r}=0.56, \mathrm{P}<0.0001$; and hemoglobin $\mathrm{r}=0.43, \mathrm{P}<0.001) . \mathrm{FC}$ : fecal calprotectin; $\mathrm{Lf}$ : lactoferrin; UCEIS: Ulcerative Colitis Endoscopic Index of Severity

Figure 1b. Correlation between ME and fecal markers

The relationship between calprotectin, lactoferrin, hemoglobin, and ME were analyzed. ME correlated significantly with calprotectin and lactoferrin levels but slightly with hemoglobin levels (calprotectin $\mathrm{r}=0.50, \mathrm{P}<0.001$; lactoferrin $\mathrm{r}=0.46, \mathrm{P}<0.001$; and hemoglobin $\mathrm{r}=0.28, \mathrm{P}=0.043)$. FC: fecal calprotectin; Lf: lactoferrin; $\mathrm{ME}$ : magnifying endoscopic stratification

Figure 2a. Receiver operating curve analysis for the fecal markers (for differentiating between UCEIS $=0$ and $\geq 1$ )

Calprotectin, at a cut-off of $201 \mathrm{\mu g} / \mathrm{ml}$, had a sensitivity of $70 \%$ and specificity of $81 \%$ for the detection of UCEIS=0 (prediction of mucosal healing) (AUC 0.76). Lactoferrin (cut-off $78.3 \mathrm{ng} / \mathrm{ml}$ ) had a sensitivity of $57 \%$ and specificity of $88 \%$ for the detection of UCEIS $=0$ (AUC 0.71). Hemoglobin (cut-off $31 \mathrm{ng} / \mathrm{ml}$ ) had a sensitivity of $64 \%$ and specificity of $88 \%$ for the detection of UCEIS=0 (AUC 0.74). FC: fecal calprotectin; Lf: lactoferrin; UCEIS: Ulcerative Colitis Endoscopic Index of Severity

Figure 2b. Receiver operating curve analysis for the fecal markers (for differentiating between $\mathrm{ME}=\mathrm{A}$ and $\geq \mathrm{B}$ )

Calprotectin, at a cut-off of $280 \mathrm{\mu g} / \mathrm{ml}$, had a sensitivity of $68 \%$ and specificity of $86 \%$ for the detection of $\mathrm{ME}=\mathrm{A}$ (prediction of mucosal healing) (AUC 0.78). Lactoferrin (cut-off $43.6 \mathrm{ng} / \mathrm{ml}$ ) had a sensitivity of $71 \%$ and specificity of $76 \%$ for the detection of $\mathrm{ME}=\mathrm{A}$ (AUC 0.77). Hemoglobin (cut-off of $34 \mathrm{ng} / \mathrm{ml}$ ) had a sensitivity of $71 \%$ and specificity of $86 \%$ for the detection of ME=A (AUC 0.78). FC: fecal calprotectin; Lf: lactoferrin; ME: magnifying endoscopic stratification 


\section{Fig.1a Correlation between UCEIS}

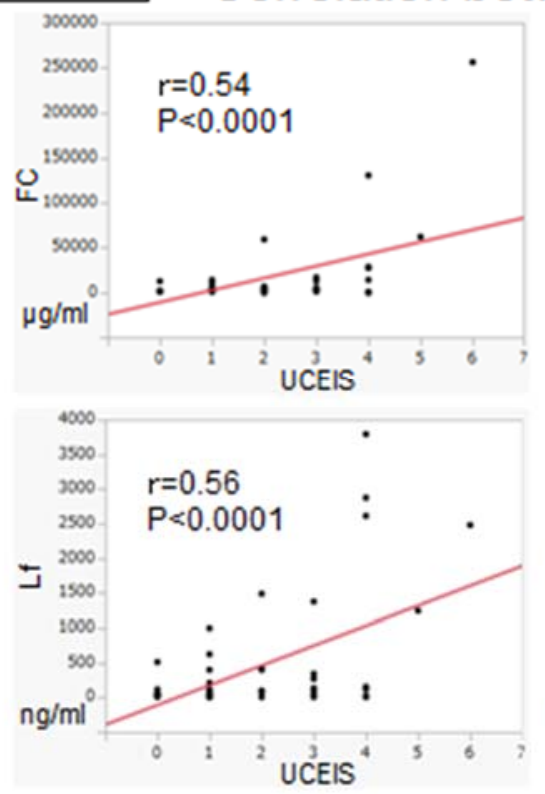

and fecal markers
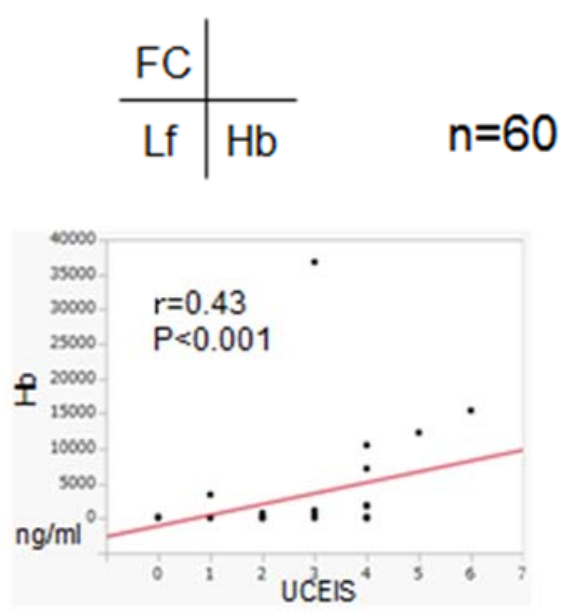

Figure 1a. Correlation between UCEIS and fecal markers

The relationships between calprotectin, lactoferrin, hemoglobin, and UCEIS were analyzed. A significant correlation was observed between UCEIS and all fecal markers (Spearman's rank correlation coefficient for calprotectin $\mathrm{r}=0.54, \mathrm{P}<0.0001$; lactoferrin $\mathrm{r}=0.56, \mathrm{P}<0.0001$; and hemoglobin $\mathrm{r}=0.43, \mathrm{P}<0.001)$. FC: fecal calprotectin; $\mathrm{Lf}$ : lactoferrin; UCEIS: Ulcerative Colitis Endoscopic Index of Severity 


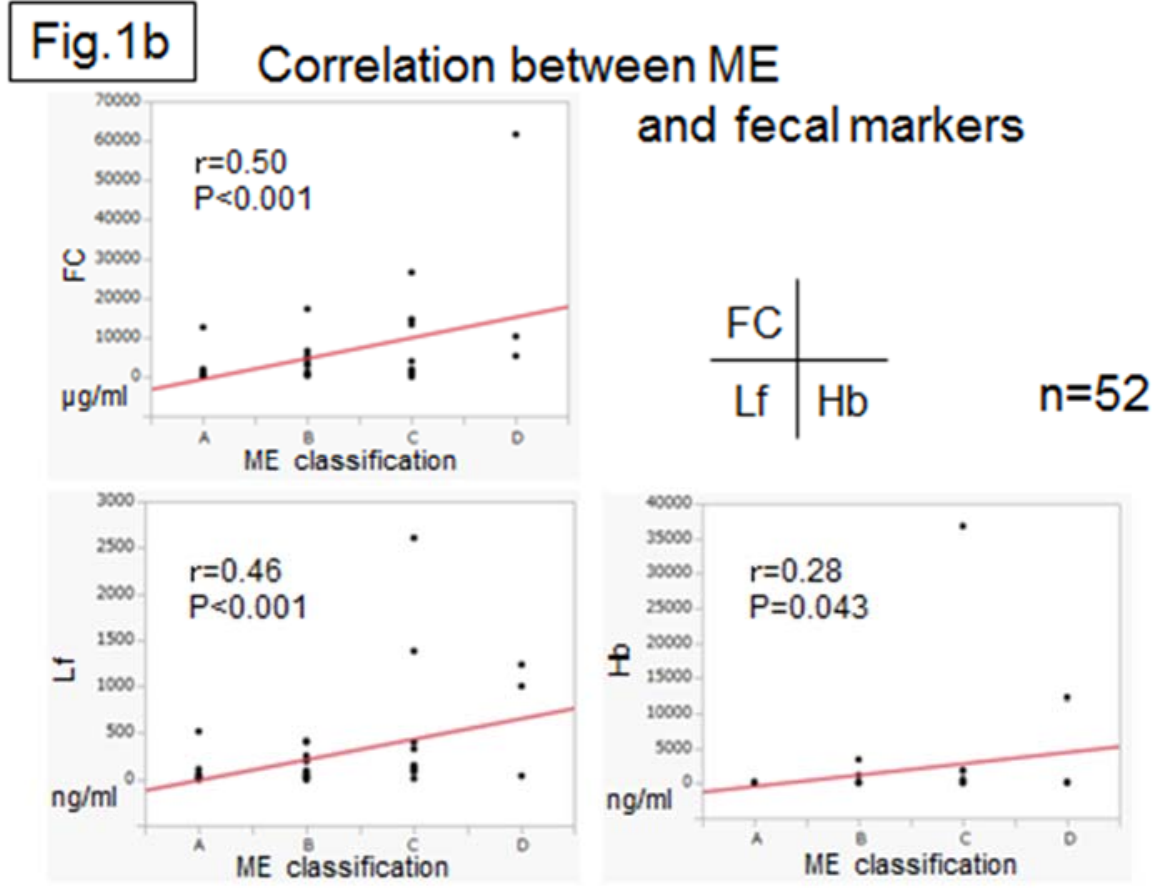

Figure 1b. Correlation between ME and fecal markers

The relationship between calprotectin, lactoferrin, hemoglobin, and ME were analyzed. ME correlated significantly with calprotectin and lactoferrin levels but slightly with hemoglobin levels (calprotectin $\mathrm{r}=0.50, \mathrm{P}<0.001$; lactoferrin $\mathrm{r}=0.46, \mathrm{P}<0.001$; and hemoglobin $\mathrm{r}=0.28, \mathrm{P}=0.043)$. $\mathrm{FC}$ : fecal calprotectin; Lf: lactoferrin; $\mathrm{ME}$ : magnifying endoscopic stratification 


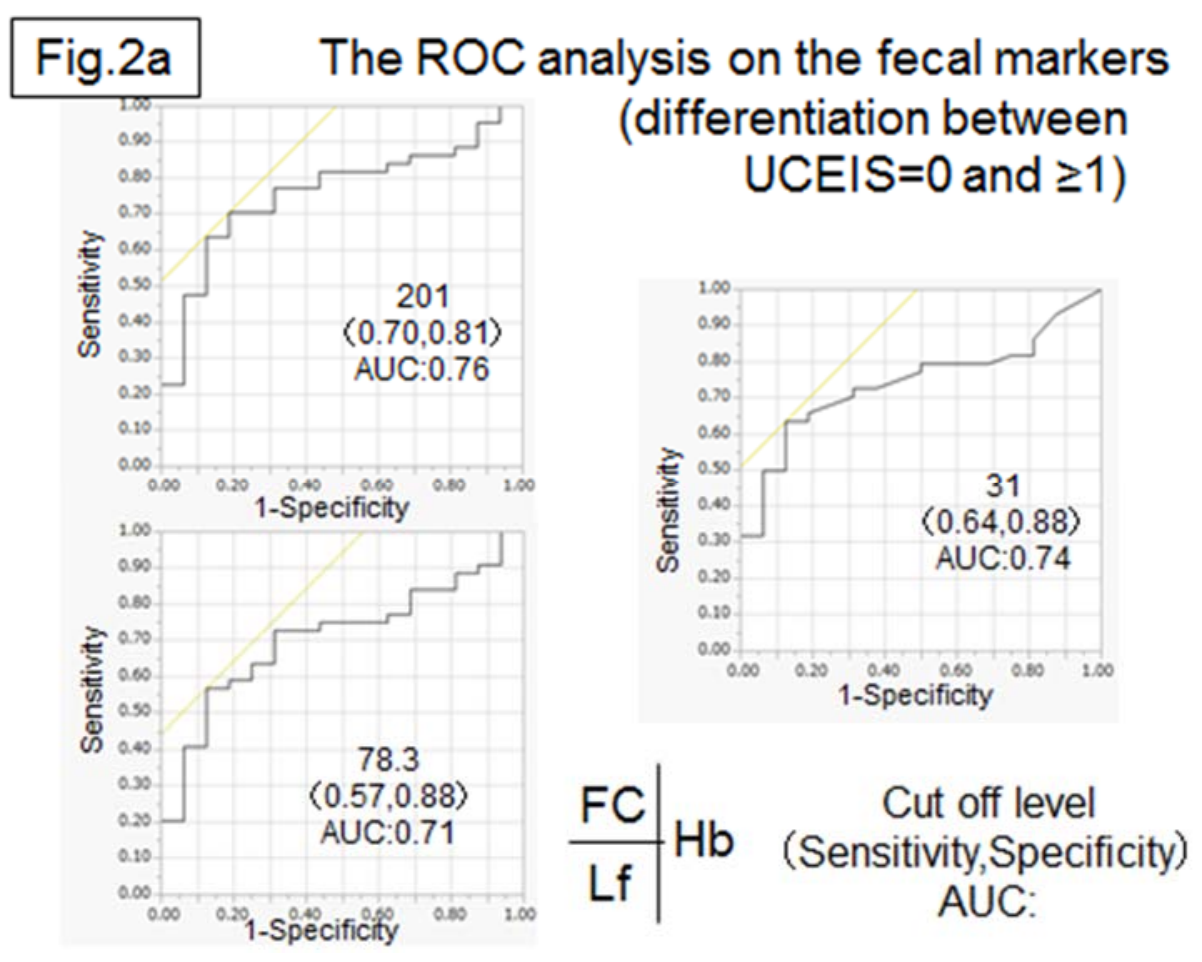

Figure 2a. Receiver operating curve analysis for the fecal markers (for differentiating between UCEIS $=0$ and $\geq 1$ )

Calprotectin, at a cut-off of $201 \mathrm{\mu g} / \mathrm{ml}$, had a sensitivity of $70 \%$ and specificity of $81 \%$ for the detection of UCEIS=0 (prediction of mucosal healing) (AUC 0.76). Lactoferrin (cut-off $78.3 \mathrm{ng} / \mathrm{ml}$ ) had a sensitivity of $57 \%$ and specificity of $88 \%$ for the detection of UCEIS $=0$ (AUC 0.71). Hemoglobin (cut-off $31 \mathrm{ng} / \mathrm{ml}$ ) had a sensitivity of $64 \%$ and specificity of $88 \%$ for the detection of UCEIS=0 (AUC 0.74). FC: fecal calprotectin; Lf: lactoferrin; UCEIS: Ulcerative Colitis Endoscopic Index of Severity 


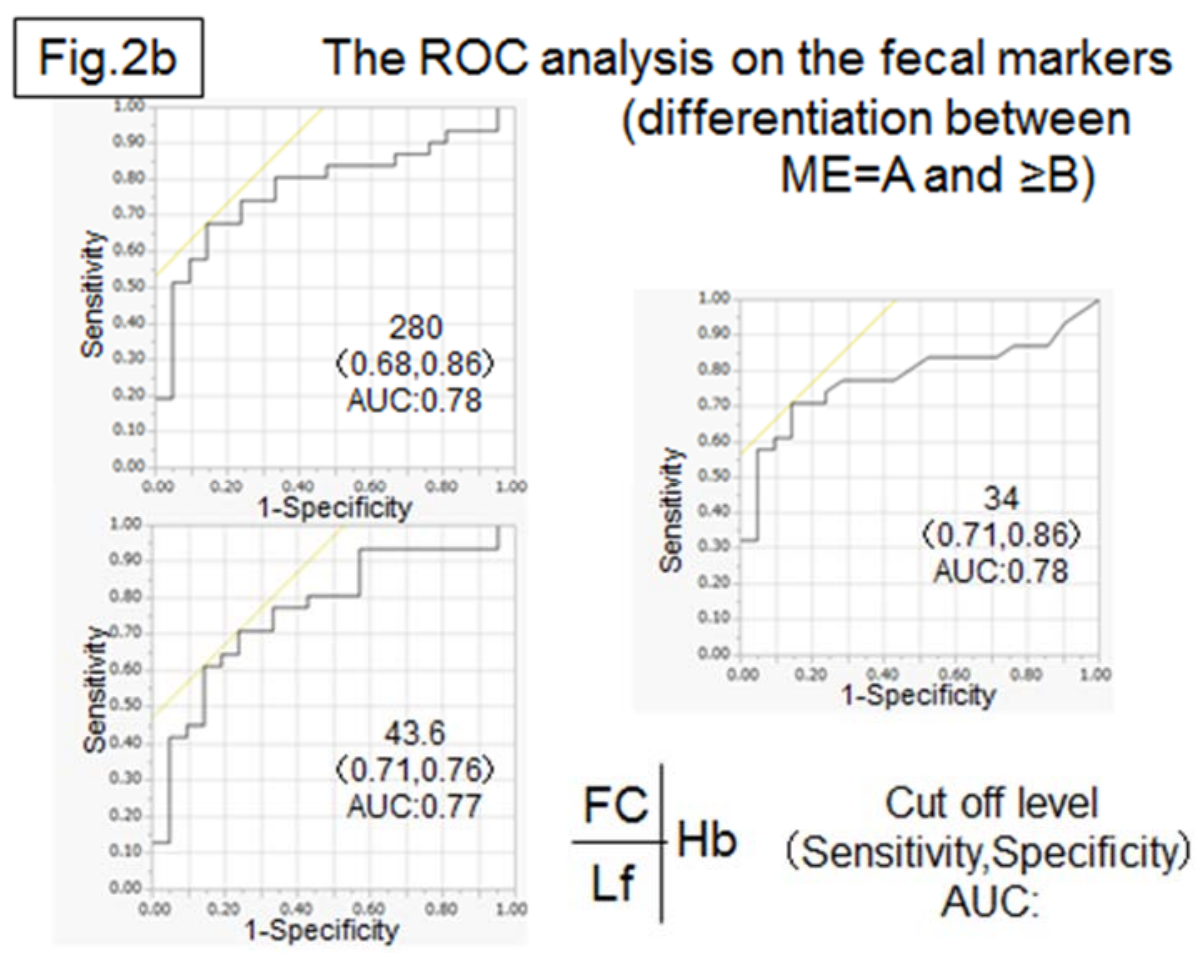

Figure 2b. Receiver operating curve analysis for the fecal markers (for differentiating between $\mathrm{ME}=\mathrm{A}$ and $\geq \mathrm{B}$ )

Calprotectin, at a cut-off of $280 \mathrm{\mu g} / \mathrm{ml}$, had a sensitivity of $68 \%$ and specificity of $86 \%$ for the detection of $\mathrm{ME}=\mathrm{A}$ (prediction of mucosal healing) (AUC 0.78). Lactoferrin (cut-off $43.6 \mathrm{ng} / \mathrm{ml})$ had a sensitivity of $71 \%$ and specificity of $76 \%$ for the detection of $\mathrm{ME}=\mathrm{A}$ (AUC 0.77). Hemoglobin (cut-off of $34 \mathrm{ng} / \mathrm{ml}$ ) had a sensitivity of $71 \%$ and specificity of $86 \%$ for the detection of $\mathrm{ME}=\mathrm{A}$ (AUC 0.78). FC: fecal calprotectin; Lf: lactoferrin; ME: magnifying endoscopic stratification 
Table 1. Characteristics of patients

\section{Patients}

Total

Age [Median (range)] years

$51(20-79)$

Gender

Male

$35(58 \%)$

Female

$25(42 \%)$

Extent of inflammation

Extensive colitis

$40(67 \%)$

Left-sided colitis

$13(22 \%)$

Proctitis

$7(12 \%)$

Concomitant medications

None

6

Salazosulfapyridine

5

Mesalamine

43

Azathioprine / Mercaptopurine

$9 / 4$

Infliximab / Adalimumab

$3 / 2$ 
Table 2. Summary of the relationship between magnifying endoscopic (ME) classifications and Ulcerative Colitis Endoscopic Index of Severity (UCEIS)

\begin{tabular}{|c|c|c|c|c|c|c|}
\hline ME-classification & $\begin{array}{c}0 \\
(\mathrm{n}=15)\end{array}$ & $\begin{array}{c}1 \\
(n=18)\end{array}$ & $\begin{array}{c}2 \\
(\mathrm{n}=6)\end{array}$ & $\begin{array}{c}3 \\
(n=8)\end{array}$ & $\begin{array}{c}4 \\
(n=4)\end{array}$ & $\begin{array}{c}5< \\
(n=1)\end{array}$ \\
\hline ME-A $\quad(n=21)$ & 15 & 6 & & & & \\
\hline ME-B $\quad(n=17)$ & & 9 & 4 & 3 & 1 & \\
\hline ME-C $\quad(n=12)$ & & 3 & 2 & 4 & 3 & \\
\hline$M E-D \quad(n=2)$ & & & & 1 & & 1 \\
\hline
\end{tabular}

A significant correlation was observed between ME and UCEIS $(r=0.74, \mathrm{P}<0.0001)$. 